\title{
Etiología y fisiopatología del SARS-CoV-2
}

\author{
Etiology and pathophysiology of SARS-CoV-2
}

\author{
Irlanda Alvarado Amador,* Jessica Bandera Anzaldo,* Laura Elena Carreto Binaghi, ${ }^{\ddagger}$ \\ Gandhi Fernando Pavón Romero, ${ }^{\S}$ Alejandro Alejandre García『

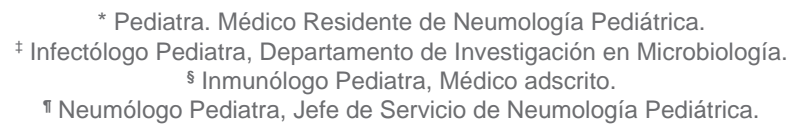

Instituto Nacional de Enfermedades Respiratorias Ismael Cosío Villegas. Ciudad de México, México.

EI SARS-CoV-2 causa una infección respiratoria aguda como en el caso de SARS-CoV y MERS-CoV, con fiebre, tos y disnea; la neumonía es una manifestación grave que puede progresar rápidamente a SDRA. ${ }^{1}$

Los coronavirus constituyen una familia de virus ARN, monocatenario y de cadena positiva, envueltos. Desde 1968, se otorga su nombre por la morfología en «corona» observada en la microscopia electrónica, donde las proyecciones de la membrana del virus, conocidas como espículas, le dan la apariencia. Pertenecen a la familia Coronaviridae, subfamilia Orthocoronaviridae, dentro del orden de los Nidovirales. ${ }^{2-4}$ Los coronavirus se dividen en tres géneros (I a III) en todos los casos de transmisión por animales. ${ }^{5}$

La subfamilia se clasifica en cuatro géneros: alfa, beta, gamma y delta, siendo los primeros dos los que infectan al humano. Se han descrito siete coronavirus que causan enfermedad en humanos: 229-E ( $\alpha$-CoV), NL63 ( $\alpha$-CoV), OC43 ( $\beta$-CoV), HKU1 $(\beta-\mathrm{CoV})$, MERS-CoV ( $\beta$-CoV), SARS-CoV $(\beta-\mathrm{CoV})$ y el séptimo miembro es el recién descubierto SARSCoV-2 ( $\beta$-CoV). ${ }^{6}$

EI SARS-CoV-2 es un virus envuelto, con un diámetro de aproximadamente 60-140 nm, cuya forma puede ser esférica, elíptica o pleomórfica. ${ }^{7} \mathrm{EI}$

Financiamiento: Ninguno.

Conflicto de intereses: Ninguno.

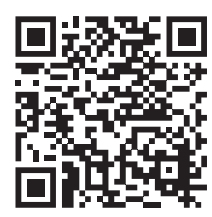

genoma viral tiene aproximadamente de 27-32 kb3 y codifica proteínas estructurales y no estructurales; por su importancia, las primeras se describen a continuación:

- Espícula (proteína S): se proyecta a través de la envoltura viral y forma las espículas de la corona; se encuentra glucosilada y es la encargada de mediar la unión del receptor, así como su fusión con la célula del huésped. ${ }^{8}$

- Proteína de membrana (M): posee dos extremos, un dominio N-terminal corto que se proyecta en la superficie externa de la envoltura y un extremo C-terminal largo interno; juega un papel importante en el ensamblaje del virus. ${ }^{9}$

- Proteína de la nucleocápside (N): se asocia con el genoma de ARN para formar la nucleocápside; se piensa que puede estar involucrada en la regulación de la síntesis del ARN e interactúa con la proteína $\mathrm{M}$ al momento de la replicación viral. ${ }^{9}$

- Proteína de la envoltura (E): es una proteína que funciona como porina, formando canales iónicos, se desconoce su función específica; sin embargo, en el virus SARS-CoV esta proteína participa en el ensamblaje del virus (Figura 1). ${ }^{10}$

Todos los coronavirus comparten algunas similitudes en la organización y expresión de su genoma, donde 16 proteínas no estructurales son

Citar como: Alvarado Al, Bandera AJ, Carreto BLE, Pavón RGF, Alejandre GA. Etiología y fisiopatología del SARS-CoV-2. Rev Latin Infect Pediatr. 2020; 33 (s1): s5-s9. https://dx.doi.org/10.35366/96667 


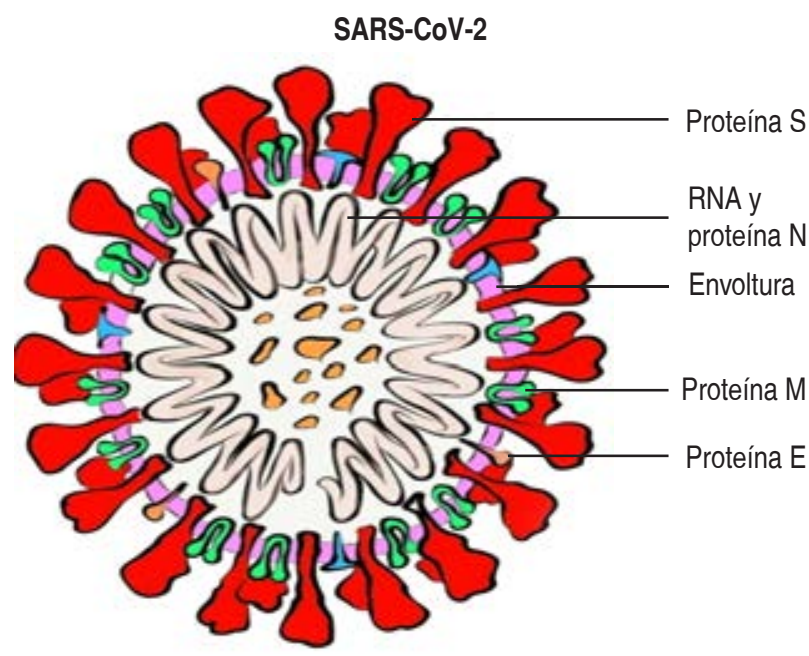

Figura 1: Esquematización del virus SARS-CoV-2. Se puede apreciar la disposición de las proteínas que componen el virión de los coronavirus humanos. (Dibujado por Alvarado Amador Osmar de Jesús).

codificadas por un marco de lectura abierto ORF1ab en el extremo 5', seguidas por proteínas estructurales codificadas por otro ORF en el extremo 3'. ${ }^{4}$

Los coronavirus son conocidos por su circulación entre algunos mamíferos y aves. ${ }^{11}$ EI SARS-CoV-2 fue aislado por primera vez en el líquido del lavado broncoalveolar (LBA) de tres pacientes con enfermedad por COVID-19 en diciembre del 2019,,$^{12,13}$ y el análisis de la secuencia genómica demostró que SARSCoV-2 es $96.2 \%$ idéntico a Bat CoV RaTG13, lo cual sugiere que el virus humano comparte similitud con el de murciélago, en relación con el mercado de Wuhan en donde inició el brote. El análisis filogenético de todo el genoma indica que SARS-CoV-2 comparte 79.5 y $50 \%$ de identidad de secuencia con SARS-CoV y MERS-CoV, respectivamente.

Aún se encuentran en estudio sus propiedades fisicoquímicas; se propone que es sensible a la radiación ultravioleta y a la exposición a altas temperaturas. Algunos estudios describen que el virus puede inactivarse a una temperatura de 56 ${ }^{\circ} \mathrm{C}$ durante media hora, y con el uso de solventes lipídicos como etanol al $75 \%$, desinfectantes con cloro, ácido peroxiacético y cloroformo; no obstante, no es sensible al uso de clorhexidina. ${ }^{14}$

El espectro de enfermedades generadas por la infección de coronavirus son principalmente respiratorias agudas y crónicas, entéricas, hematológicas y del sistema nervioso central. ${ }^{5}$ Los tipos de virus $\beta$-CoV, SARS-CoV y MERS-CoV generan infecciones potencialmente graves para el sistema respiratorio. ${ }^{12}$
El mecanismo de transmisión de la enfermedad por SARS-CoV-2 es de persona a persona por medio de la vía aérea a través de las gotas de Flügge que se exhalan al toser, estornudar o hablar y son inhaladas o depositadas en boca y conjuntivas oculares, así como superficies, que pueden fungir como fómites. Informes recientes indican que el SARS-CoV-2 se puede detectar en la orina y las heces de pacientes confirmados, lo que implica un riesgo de transmisión fecal-oral (Figura 2). Aún no se ha documentado la transmisión materno-fetal. El periodo de incubación en promedio es de 5.2 días con una media de 4.7 días que transcurren entre el inicio de los síntomas. ${ }^{15}$

Factores virales y del huésped influyen en la patogénesis del SARS-CoV-2. La ACE 2 es una proteína de membrana tipo I que tiene receptores en el pulmón, corazón, riñón e intestino, principalmente asociados con enfermedades cardiovasculares. Se ha documentado que la replicación viral primaria ocurre en el epitelio de la mucosa de la cavidad nasal y faringe. Los receptores ACE 2 que están localizados en el tracto respiratorio inferior de los humanos son los receptores celulares para SARSCoV-2, ya que el virión cuenta con S-glucoproteína en la superficie del coronavirus que es capaz de unirse al receptor ACE 2 de las células humanas. La glucoproteína S incluye dos subunidades, S1 y S2: la primera determina el tropismo celular, y la segunda media la fusión de la membrana celular del virus. Posterior a esta fusión de membrana, el ARN del genoma viral es liberado en el citoplasma, el ARN no envuelto traduce dos lipoproteínas pp1a y pp1ab, que forman el RTC en una vesícula de doble membrana que continuamente se replica. ${ }^{15}$

La evidencia biofísica y estructural sugiere que la proteína S del SARS-CoV-2 probablemente se une al ACE 2 humano con una capacidad 10 a 20 veces mayor que el SARS-CoV que influye en su gravedad. Cuando la enfermedad progresa, ocasiona SDRA que representa la causa de mayor mortalidad en los trastornos respiratorios agudos. Literatura reciente describe la relación de la susceptibilidad genética y la inflamación, ya que no todas las personas expuestas a SARS-CoV-2 están infectadas y no todos los pacientes infectados desarrollan enfermedad grave. ${ }^{15,16}$

Yufang Shi y colaboradore ${ }^{16}$ dividen la enfermedad en tres etapas, las cuales hemos discutido y modificado para enriquecerlas con base en los reportes.

La Tabla 1 muestra las etapas de la enfermedad por SARS-CoV-2 en humanos. 
Rev Latin Infect Pediatr 2020; 33 (s1): s5-s9

Aún no se puede explicar del todo el amplio espectro de la enfermedad, durante la fase 1 y 2 la respuesta inmune adaptativa es requerida para la eliminación del virus y prevenir la progresión de la enfermedad. Existen diferencias genéticas que parecen contribuir en variaciones con la respuesta inmune ante los patógenos. En la etapa 3, el SLC genera importante daño pulmonar. El mal estado general del huésped y la presencia de comorbilidades facilitan la propagación del virus y el tropismo por los órganos diana con receptores ACE 2, así como la producción aumentada de IL-6, IL-1 y TNF- $\alpha$ en casos graves. ${ }^{16,17}$ En resumen, la enfermedad grave se caracteriza por neumonía, linfopenia y SLC, que activan una respuesta inmune exagerada que genera daño a nivel local y sistémico. ${ }^{18} \mathrm{~A}$ continuación describiremos el mecanismo inmunológico que se desarrolla en el paciente con COVID-19:

Una vez que el SARS-COV-2 accede a las células y subsecuentemente libera su material genético (ARN), es reconocido por receptores de la inmunidad innata localizados de manera intracelular, como el receptor tipo Toll 7 (TLR7), RIG-1 y MDA 5 , activando una cascada de señalización, lo que conduce a la expresión de IFN tipo I ( $\alpha$ y $\beta$ ) cuyo objetivo es interferir en la replicación viral. Por otra parte, los antígenos virales pueden ser procesados por las células presentadoras de antígeno mediante su MHC-I al TCR del linfocito T CD8 ${ }^{+}$, lo cual conlleva la liberación de sus enzimas proteolíticas (citotoxicidad). Al mismo tiempo, comienza la síntesis incrementada de mediadores proinflamatorios (tormenta de citocinas) como: IL-1B (activación de neutrófilos y pirógeno endógeno), IL-6 (activación de neutrófilos), IL-7 (diferenciación de linfocitos T), IL-8 (activación de neutrófilos), IL-9 (factor de crecimiento para linfocitos), IL-10 (suprime la proliferación y producción de citocinas de linfocitos) y TNF- $\alpha$ (activa la respuesta de neutrófilos e incrementa la síntesis de PCR). ${ }^{19}$
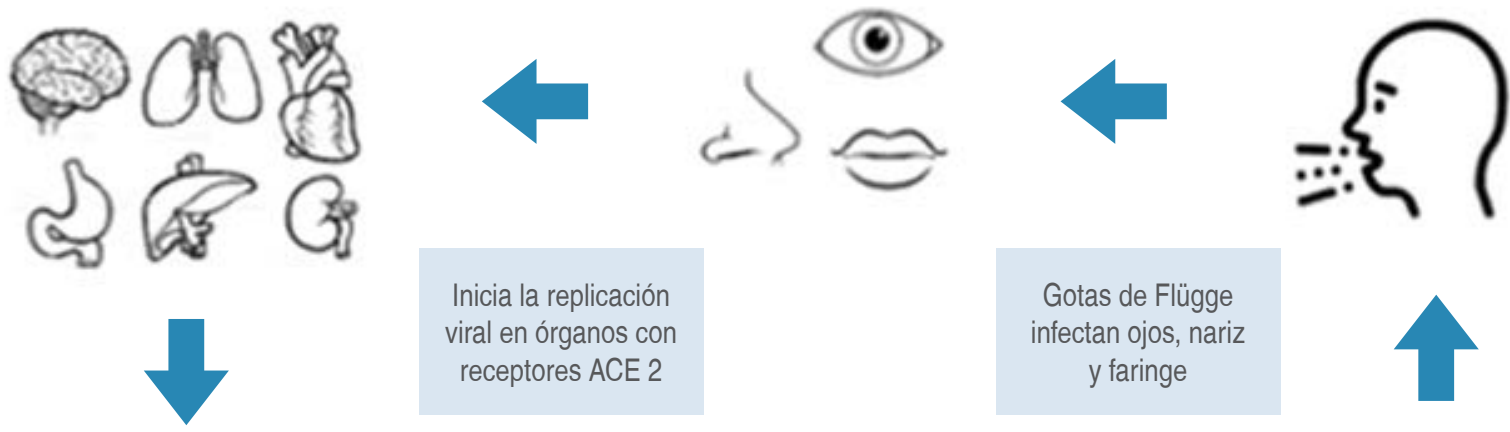

Inicia la replicación viral en órganos con receptores ACE 2

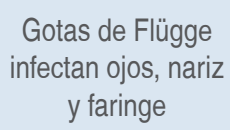

y faringe

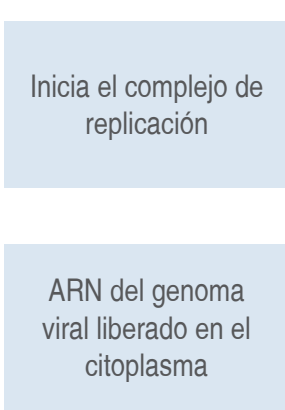

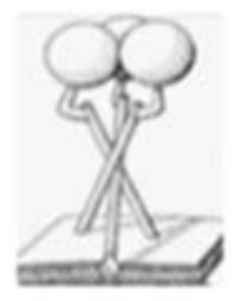

Glucoproteína S, subunidades S1 (tropismo celular) y S2 (fusión de las membranas)
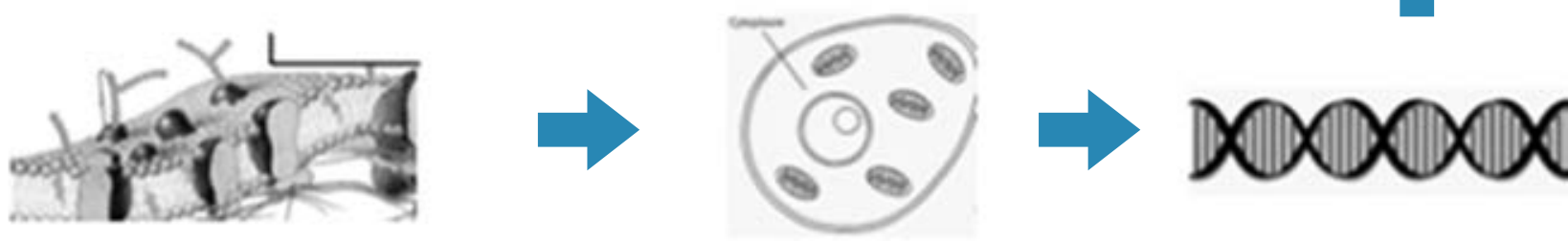

Figura 2: Diagrama de la fisiopatología del COVID-19. 


\begin{tabular}{|c|c|c|c|}
\hline Etapa 1 & Etapa 2 & Etapa 3 & Etapa 4 \\
\hline Periodo de incubación & Periodo de contagiosidad & Periodo de contagiosidad & Periodo de resolución \\
\hline Paciente asintomático & Paciente sintomático no grave & $\begin{array}{l}\text { Paciente sintomático, con deterioro } \\
\text { respiratorio grave }\end{array}$ & Paciente asintomático \\
\hline Con o sin virus detectable & Con virus detectable & Con virus detectable con alta carga & Con o sin virus detectable \\
\hline
\end{tabular}

Respecto a la inmunidad celular, se ha demostrado que los pacientes con enfermedad grave padecen linfopenia $\left(\leq 600 \mathrm{cel} / \mathrm{mm}^{3}\right)$, hasta ahora la evidencia sugiere una mayor participación de los linfocitos T citotóxicos (CD8+) en la etapa aguda; en etapas avanzadas, cuando se presenta coinfección existe aumento de los leucocitos. Un aumento en el recuento de neutrófilos, así como el incremento del ratio neutrófilos/linfocitos, generalmente indica una mayor gravedad de la enfermedad y desenlace poco favorable. En cuanto a la inmunidad humoral, se ha descrito que en el plasma de pacientes convalecientes, las células B producen anticuerpos dirigidos a la glucoproteína SARS-CoV-2, específicamente a la proteína S. Xueto CA y colaboradores refieren que en los pacientes con enfermedad grave existen mayores niveles de lgG y títulos más altos de anticuerpos totales asociados con peor pronóstico. ${ }^{18}$

La tormenta de citocinas es el factor que genera el daño al epitelio respiratorio. Interesantemente, se ha demostrado que los pacientes que requieren cuidados intensivos presentan aumento muy importante de IL-6 y GM-CSF. Estudios histopatológicos relatan como hallazgo infiltración masiva de neutrófilos y macrófagos, daño alveolar difuso con formación de membranas hialinas y engrosamiento de la pared alveolar y necrosis de ganglios linfáticos sugerente de daño inmunomediado. ${ }^{18}$

Los haplotipos HLA se asocian con distintas susceptibilidades a la enfermedad, por lo que se siguen estudiando los loci HLA específicos asociados con la inmunidad anti-SARS-CoV-2. Debido a la emergencia sanitaria ante una enfermedad nueva, continuamente se estudia y se agregan cambios a la literatura actual con nuevos conocimientos.

\section{REFERENCIAS}

1. Ye ZW, Yuan S, Yuen KS, Fung SY, Chan CP, Jin DY. Zoonotic origins of human coronaviruses. Int J Biol Sci. 2020; 16 (10): 1686-1697.
2. Chan JFW, Lau SKP, To KKW, Cheng VCC, Woo PCY, Yue KY. Middle east respiratory syndrome coronavirus: another zoonotic betacoronavirus causing SARS-like disease. Clin Microbiol Rev. 2015; 28 (2): 465-522.

3. Shanmugaraj B, Siriwattananon K, Wangkanont K, Phoolcharoen W. Perspectives on monoclonal antibody therapy as potential therapeutic intervention for Coronavirus disease-19 (COVID-19). Asian Pac J Allergy Immunol. 2020; 38 (1): 10-18

4. Su S, Wong G, Shi W, Liu J, Lai ACK, Zhou J et al. Epidemiology, genetic recombination, and pathogenesis of coronaviruses. Trends Microbiol. 2016; 24 (6): 490-502.

5. Weiss SR, Navas-Martin S. Coronavirus pathogenesis and the emerging pathogen severe acute respiratory syndrome coronavirus. Microbiol Mol Biol Rev [Internet]. 2005; 69 (4): 635-664. Available from: https://www.ncbi.nlm.nih.gov/pmc/ articles/PMC1306801/pdf/0007-05.pdf

6. Qiang XL, Xu P, Fang G, Liu W Bin, Kou Z. Using the spike protein feature to predict infection risk and monitor the evolutionary dynamic of coronavirus. Infect Dis poverty. 2020; 9 (1): 33.

7. Calvo C, García López-Hortelano M, de Carlos Vicente JC, Vázquez Martínez JL; Grupo de trabajo de la Asociación Española de Pediatría para el brote de infección por Coronavirus, colaboradores con el Ministerio de Sanidad; Miembros del Grupo de Expertos de la AEP. Recomendaciones sobre el manejo clínico de la infección por el «nuevo coronavirus» SARS-CoV2. Grupo de trabajo de la Asociación Española de Pediatría (AEP). An Pediatr (Barc). 2020; 92 (4): 241.e1-241.e11.

8. Enjuanes L, Smerdou C, Castilla J, Anton IM, Torres JM, Sola I et al. Development of protection against coronavirus induced diseases: a review. Adv Exp Med Biol. 1995; 380: 197-211.

9. Masters PS, Kuo L, Ye R, Hurst KR, Koetzner CA, Hsue B. Genetic and molecular biological analysis of protein-protein interactions in coronavirus assembly. Adv Exp Med Biol. 2006; 581: 163-173.

10. Siu YL, Teoh KT, Lo J, Chan CM, Kien F, Escriou N et al. The $\mathrm{M}, \mathrm{E}$, and $\mathrm{N}$ structural proteins of the severe acute respiratory syndrome coronavirus are required for efficient assembly, trafficking, and release of virus-like particles. J Virol. 2008; 82 (22): 11318-11330.

11. Li X, Zai J, Zhao Q, Nie Q, Li Y, Foley BT et al. Evolutionary history, potential intermediate animal host, and crossspecies analyses of SARS-CoV-2. J Med Virol. 2020; 92 (6): 602-611.

12. Lai CC, Liu YH, Wang CY, Wang YH, Hsueh SC, Yen MY et al. Asymptomatic carrier state, acute respiratory disease, and pneumonia due to severe acute respiratory syndrome coronavirus 2 (SARS-CoV-2): facts and myths. J Microbiol 
Rev Latin Infect Pediatr 2020; 33 (s1): s5-s9

Immunol Infect. 2020; 53 (3): 404-412. doi: 10.1016/j. jmii.2020.02.012

13. Jin $Y$, Yang $\mathrm{H}$, Ji W, Wu W, Chen S, Zhang W et al. Virology, epidemiology, pathogenesis, and control of COVID-19. Viruses [Internet]. Viruses. 2020; 12 (4): 372. Available from: https://www.mdpi.com/1999-4915/12/4/372

14. Alyami M. Guidance for Corona Virus Disease 2019 [Internet]. 2020. 4-5, 42-43 p. Available from: https://www. uoa.gr/fileadmin/user_upload/PDF-files/anakoinwseis/ themata_ygeias/1603_Guidance_for_Corona_Virus_ Disease_2019.pdf

15. Guo YR, Cao QD, Hong ZS, Tan YY, Chen SD, Jin HJ et al. The origin, transmission and clinical therapies on coronavirus disease 2019 (COVID-19) outbreak - an update on the status. Mil Med Res [Internet]. 2020; 7 (1): 11. Available from: https://mmrjournal.biomedcentral.com/track/ pdf/10.1186/s40779-020-00240-0

16. Shi $Y$, Wang $Y$, Shao C, Huang J, Gan J, Huang $X$ et al. COVID-19 infection: the perspectives on immune responses.
Cell Death Differ [Internet]. 2020; 27 (5): 1451-1454. Available from: http://dx.doi.org/10.1038/s41418-020-0530-3

17. Guan W, Ni Z, Hu Y, Liang W, Ou C, He J et al. Clinical characteristics of coronavirus disease 2019 in China. N Engl $J$ Med [Internet]. 2020; 382 (18): 1708-1720. Available from: https://www.nejm.org/doi/pdf/10.1056/nejmoa2002032

18. Cao X. COVID-19: immunopathology and its implications for therapy. Nat Rev Immunol [Internet]. 2020; 20 (5): 269-270. Available from: http://www.ncbi.nlm.nih.gov/ pubmed/32273594

19. Nikolich-Zugich J, Knox KS, Rios CT, Natt B, Bhattacharya D, Fain MJ. SARS-CoV-2 and COVID-19 in older adults: what we may expect regarding pathogenesis, immune responses, and outcomes. Geroscience [Internet]. 2020; 42 (2): 505-514. Available from: http://www.ncbi.nlm.nih.gov/pubmed/32274617

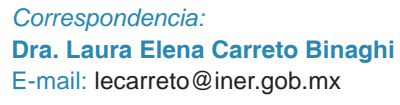

